\title{
Los experimentos discrepantes como instrumento mediador en el desarrollo de la intuición física
}

The discrepant experiment in the development of physical intuition

Luis H. Barbosa

Facultad de Ingeniería, Grupo Física y Matemática, Depto de Ciencias Naturales, Universidad Central. Centro de Investigación en Ciencia Aplicada y Tecnología Avanzada, Instituto Politécnico Nacional, Av. Legaria 694, Col. Irrigación, C. P. 11500, México D. F.

\section{Resumen}

Los Experimentos Discrepantes (ExD) son montajes que al accionarlos generan una fenomenología contra-intuitiva que puede ser utilizada favorablemente en la enseñanza de la física. Se ilustran algunas reflexiones sobre los escenarios que pueden implementar estos montajes, así como sus fortalezas y debilidades. Una experiencia de cerca de tres años, con estudiantes de ingeniería, nos ha mostrado que los ExD permiten un escenario fértil que moviliza al estudioso a ejercitar su capacidad de observación, a desarrollar su creatividad y a mejorar su intuición física. Además, lo entrena en actividades propias de la investigación en ciencias como conjeturar, argumentar, abstraer, modelar y socializar conocimiento en forma verbal y escrita. Se presentan resultados de investigación sobre el uso de ExD en el desarrollo de la intuición física en estudiantes de ingeniería.

\section{Palabras clave}

Demostraciones experimentales, experimentos discrepantes, Física Educativa.

\section{Abstract}

The discrepant experiments (ExD) are assemblies that are operable to generate a counter intuitive phenomenon that can be used favorably in the teaching of physics. We illustrate some thoughts on the scenarios that can deploy these assemblies, as well as their strengths and weaknesses. Our experience of nearly three years, with engineering students, has shown us that a scene bearing ExD allow the student to move to exercise its monitoring capability, develop their creativity and improve their physical intuition. The students were trained in activities of research in science and to conjecture, to arguably, to abstraction, to modeling and socialize knowledge orally and in writing. We present results of research on the use of EXD in developing the physical intuition in engineering students.

\section{Keywords}

Discrepant events, discrepant experiments, science inquiry, experimental demonstration.

\section{Introducción}

Hace poco me encontraba en el supermercado comprando un aguacate para el almuerzo. Mi esposa me dijo que yo lo escogiera ya que tenía más intuición para escoger el fruto apropiado. Revisé, y entre varios, escogí uno que luego de abrirlo en casa, efectivamente resultó ser el esperado: blando y de buen sabor. Muchas de las actividades de nuestra diaria involucran un tinte de esto que llamamos intuición que resulta en gran parte del sentido que damos a la experiencia mediante el reconocimiento de patrones y la representación de estos a través de modelos. A este 
tipo de intuición es a la que quiero referime como el potencial que mediante experimentos discrepantes de física se puede cultivar y desarrollar en estudiantes.

La intuición física es la habilidad de una persona para distinguir lo relevante y despreciar aquello que no lo es en una cierta situación o problema. Por tanto, es la habilidad que utilizamos para representar la estructura de los eventos y sistemas físicos. Dice (Krauss, 1996) que aunque puedan requerirse estudios superiores para dominar la Física, no se necesita un tomo masivo para elucidarla, pues a todo nivel de esta área el físico utiliza unas cuantas herramientas con las que enfoca sus búsquedas, su investigación: estimación, análisis dimensional, simetrías, metáforas, analogías y modelos, entre otras.

La habilidad con que la gente usa metáforas, analogías y modelos (Hestenes, 2006), es un componente importante de su intuición y su conocimiento. Utilizamos las metáforas en la vida cotidiana de pensamiento y de expresión mucho más de lo que estamos conscientes. Por supuesto, el propósito es orientar a los estudiantes a reconstruir sus metáforas de manera que coincidan con la intuición física del consenso cientúfico, así como sus modelos a partir de analogías que den sentido a la fenomenología y conocimiento de la física que aprende en su formación.

En trabajos anteriores (Barbosa, 2008; Barbosa, Talero, 2009) se ha mostrado que los experimentos discrepantes (ExD) pueden generar un escenario propicio para un aprendizaje activo de la física donde el estudiante puede cultivar y desarrollar esas herramientas que afinan su intuición física.

A continuación se presenta las bondades de una metodología mediada con ExD en el desarrollo de habilidades investigativas del estudiante. En la sección III se ilustra cierta experiencia con ExD en el aprendizaje de la Física y finalmente en la sección IV se presentan las condusiones.

\section{Los experimentos discrepantes en una formación investigativa de estudiantes}

Uno de los tantos problemas de la metodología tradicional es que debido al afán por cumplir con un plan curricular, no hay tiempo para escenarios donde se genere gusto y actitud, por el asombro, por la observación, por la pregunta, por la conjetura, por el análisis, etc. Como manifiesta (Barrera, 2007), es necesario, pues crear estrategias de aprendizaje autónomas que permitan alcanzar el objetivo de "aprender a aprender". Particularmente, los EXD generan un escenario propicio para que el estudiante se entrene en acciones propias de la investigación (Barbosa, 2009) como observación, conjeturación, abstracción, predicción, modelación, etc.

Un ExD es un montaje que al accionarlo manifiesta un fenómeno impactante o contraintuitivo para el estudiante. El fenómeno puede corresponder a un suceso que ocurre cuando el observador está esperando otro. De otra manera se puede decir que un ExD exhibe una fenomenología sorpresiva, inesperada, paradójica y que ofende la intuición de quien lo observa.

Uno de los tantos problemas de la metodología tradicional es que debido al afán por cumplir con un plan curricular, no hay tiempo para escenarios donde se genere gusto y actitud, por el asombro, por la observación, por la pregunta, por la conjetura, por el análisis, etc. Como manifiesta (Barrera, 2007), es necesario, pues crear estrategias de aprendizaje autónomas que permitan alcanzar el objetivo de "aprender a aprender". Particularmente, los ExD generan un escenario propicio para que el estudiante se entrene en acciones propias de la investigación (Barbosa, 2009) como observación, conjeturación, abstracción, predicción, modelación, etc. 
Un EXD es un montaje que al accionarlo manifiesta un fenómeno impactante o contraintuitivo para el estudiante. El fenómeno puede corresponder a un suceso que ocurre cuando el observador está esperando otro. De otra manera se puede decir que un ExD exhibe una fenomenología sorpresiva, inesperada, paradójica y que ofende la intuición de quien lo observa.

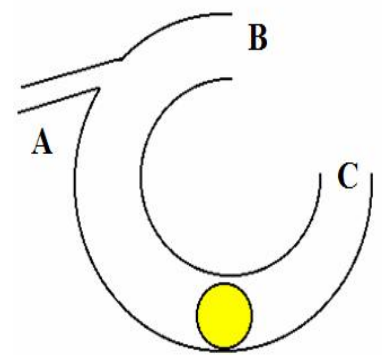

Figura No. 1. El soplador mágico.

Por ejemplo, la esfera en el soplador sale por la boca B del tubo (Barbosa, 2003), el trompo se voltea (Barbosa, 2008). Si el evento es impactante también genera desequilibrio y por tanto atrapa al estudiante. El desequilibrio ya no es entre el suceso y lo esperado sino porque el estudiante no tiene presaberes inmediatos que le permitan entender el fenómeno.

La discrepancia generada por los ExD como motor de aprendizaje mediante esta metodología tiene soporte en la teoría de la disonancia (Festinger, 1957). Él afirma que una persona presenta disonancia cognitiva, cuando percibe dos informaciones contrarias de un mismo estimulo o mantiene al mismo tiempo dos pensamientos que están en conflicto. Es decir, presenta incompatibilidad de dos cogniciones simultáneas.

Festinger plantea que al producirse esa incongruencia o disonancia de manera muy apreciable, la persona se ve automáticamente motivada para esforzarse en generar ideas y creencias nuevas que permitan reducir la tensión hasta conseguir que el conjunto de sus ideas y actitudes encajen entre sí, logrando una cierta coherencia intema.

El modo en que se produce la reducción de la disonancia puede involucrar distintos caminos. Por ejemplo, un cambio de actitud o de ideas ante la realidad. Por supuesto, como el fenómeno del montaje no se puede cambiar, físicamente, el único camino que queda, para reducir el desequilibrio, es cambiar de ideas. Aquí es donde el maestro de Física, no debe apresurarse a dar una respuesta sino que al unísono con sus estudiantes debe procurar jugar, degustar el fenómeno, hacer preguntas y sobre todo manifestar al estudiante que se inicia una empresa ardua para develar el cómo y el por qué del fenómeno.

Como la tarea es reducir la disonancia, resulta apropiado asignar unas tareas para el éxito de esa gran empresa. Es importante sugerir alguna metodología de trabajo para observar, anotar detalles, plantear preguntas, generar conjeturas, utilizar algún modelo conceptual y cadenas de razonamiento para describir el fenómeno; acordar momentos para presentar avances del entendimiento del evento, búsqueda de información, etc.

Muy importante en esta metodología es conocer el rol del experimento en la Física y en la enseñanza de la Física ya que provee el alcance y posibilita los distintos tipos de experimentos en la práctica educativa. Según (Etkina y Otros, 2002), es posible encontrar tres tipos de experimentos dentro de la Física: los experimentos observacionales, los experimentos que prueban o refitan un modelo teórico y los experimentos de aplicaciones. Un experimento observacional corresponde a aquel cuando los físicos estudian un fenómeno desconocido. Estos ayudan a desarrollar un nuevo modelo, por ejemplo, las observaciones de los gases en el siglo XVII, o de los espectros en el siglo XIX, o de las observaciones de Becquerel sobre radiactividad. Los experimentos de prueba sirven 
para verificar o refutar una cierta hipótesis o idea. Por ejemplo, los experimentos de Hertz probaron las predicciones de ondas electromagnéticas de Maxwell. El experimento de Stern-Gerlach probó la idea de cuantización de espín. Un experimento de aplicación puede corresponder a la exploración de un satélite o cometa. En la enseñanza de la Física, Etkina y otros precisan que los experimentos pueden dasificarse de acuerdo al objetivo del experimento: los experimentos observacionales, los experimentos de prueba y los experimentos de aplicación. Experimentos observacionales tienen objetivo observar un nuevo fenómeno. Más tarde los estudiantes explican sus observaciones. Los experimentos de prueba tienen como objetivo refutar o verificar las explicaciones inventadas de fenómenos trabajados o hacer predicciones de un nuevo experimento. Los experimentos de aplicación tienen como objetivo explicar lo que fue probado en el tipo dos de experimento, explicar un nuevo fenómeno o diseñar una técnica. Los profesores ayudan a los estudiantes a diferenciar entre evidencias observacionales e inferencias. Los estudiantes aprenden a probar inferencias experimentalmente y a mirar la aplicabilidad de sus ideas. Entonces, ellos adquieren destrezas cientúficas.

Un aspecto importante de los ExD es que pueden potenciar el trabajo en grupo. En realidad, yo insisto a los estudiantes a que accionen, charlen, anoten lo que los sorprenda, sus interrogantes, sus dificultades, sus conjeturas, etc. Al conformar grupos de trabajo, se observa que el estudiante debe esforzarse a emitir sus razonamientos y a expresar ideas coherentes para sus compañeros que escuchan. Se nota que esto los hace crecer en su discurso. Como expresa (Redish y Otros, 1997, 1998), el aprendizaje es más efectivo a través de las interacciones sociales, de otra manera, la interacción entre pares actúa como una herramienta de enorme valor pedagógico. Por eso la constante es invitarlos a que se dejen absorber por el fenómeno, a que giren alrededor del mismo. A la postre se consigue que efectivamente los estudiantes giren en tomo al fenómeno, y fundamentalmente, se logra que se concentren en el problema de la descripción y de las causas del fenómeno. Se observa que explicar, describir e innovar se convierte en una obsesión, en un reto, en una pasión.

\section{Experiencia de los ExD en el desarrollo de la intuición física en estudiantes}

Para este estudio se utilizaron como ExD el soplador mágico (Barbosa, 2003) y la compuerta mágica (Barbosa, Talero, 2009). Estos montajes fueron utilizados altemativamente a la clase tradicional con 98 estudiantes de tres grupos. Se presentó el montaje con mucha emotividad como recomienda (Liem, 1991). Se realizó una encuesta diagnóstica acerca de lo que debía esperar el estudiante, y sin precisar el fenómeno que debía ocurrir, luego se accionó; muchos incrédulos pedían repetirlo otra vez. Algunos dijeron que había algún truco de magia por lo que se les entregó un prototipo para accionar. Inmediatamente, ellos pidieron explicación. Por experiencia, no describí nada. Mejor inicié con la siguiente enseñanza: Les contaré una plática entre el maestro y el disćpulo auando este último objeta a su maestro: iGran sabio!, Tu siempre nos cuentas parábolas pero nunca nos das el significado; y el maestro contestó, iPequeño saltamontes!, cite gustaría que te diera una manzana ya masticada? Ante esta parábola, los estudiantes cambiaron su actitud y pidieron tiempo para accionar, observar, pensar y describir para un momento posterior. Algunos jugaron, otros comenzaron a conjeturar. Para ese momento, un monitor se encargaba de registrar, preguntas, conjeturas, cantidad de intervenciones, etc. Inmediatamente, se les entregó un material escrito con algunas instrucciones para trabajo posterior. Acordamos hablar del asunto, 8 días después. Sin embargo, durante la semana los estudiantes me abordaron con mucha frecuencia para intentar dar explicación. Hubo cinco momentos de interacción de 60 minutos para cada montaje, y contrario a la metodología tradicional, muchos estudiantes participaban con bastante motivación. 
Los talleres de trabajo utilizados presentan un esquema general que se puede resumir en cuatro etapas: ( $($ ) Familiarización con el montaje: A veces se construye (compuerta), siempre se juega, se degusta el fenómeno, se observa. En otras, se manda construir (soplador) y se hacen cambios para que funcione. (ii) Obtención de información: Se formulan interrogantes, conjeturas, se identifica la discrepancia, se describe el hecho, se identifica lo relevante e irrelevante. (iit) Abstracción y descripción: Mediante modelos conceptuales se descibe el fenómeno, se identifican posibles variables que podrían medirse, se aíslan aspectos del fenómeno y se mide, se estudia la relación entre variables medidas, se abstrae, se modela y se verifica. (iv) Socialización de la actividad: Se expone, se debate, se presentan las hipótesis, resultados, etc.

En la etapa de descripción del fenómeno se hizo énfasis en la importancia de la base de conocimiento ya acumulada, sin olvidar que se pueden inventar imágenes y modelos basados en las ideas de Hertz (Heisenberg, 1975), "elaboramos imágenes aparentes ó símbolos de los objetos exteriores tal que: las conseauencias lógicas de la imagen son a su vez imágenes de las conseauencias naturales de los objetos representados. Las imágenes como los modelos presentan ciertas ventajas: Podemos desarrollar rápidamente las consecuencias que el mundo exterior nos sacará a la luz más que lentamente ó como resultado de nuestra intervención. Podemos adelantarnos a nuestros hechos y tomar nuestras decisiones actuales de acuerdo con nuestro conocimiento alcanzado". Para Hertz estas deben ser las características de una imagen:

> Debe cumplir la condición fundamental, es decir, las consecuencias lógicas de la imagen son a su vez imágenes de las consecuencias naturales de los objetos representados.

$>$ Debe ser legítima, es decir que no hayan ni términos ni definiciones contradictorias a las leyes de nuestro pensamiento.

Debe ser adecuada, es decir, coherente con las cosas externas.

$>$ Debe ser precisa, es decir, de dos imágenes dadas, será más precisa aquella que refleje más aspectos esenciales.

$>$ Debe ser manejable, es decir, de dos imágenes dadas, será más manejable aquella que incluya menos notas superfluas.

Se hizo un trabajo de campo con estudiantes de tercer semestre de ingeniería en la asignatura de física mecánica. No se empleó grupo control. El referente de comparación se hizo midiendo indicadores de motivación, cantidad de preguntas, argumentación, etc., en cinco momentos para cada montaje, con tres grupos de semestres diferentes en 2006 y 2007. Inicialmente se utilizó el método tradicional para incorporar cinemática del plano y dinámica. Posteriormente en los mismos grupos, se incorporó fluidos con clases expositivas cortas y el uso del soplador y la compuerta monitoreando con los mismos indicadores de la metodología inicial. Con el método tradicional se registran datos de muy bajo desempeño de los estudiantes. En contraste, con experimentos discrepantes se registran datos de alta motivación, interrogación, argumentación, atención de observación, búsqueda de información y aprendizaje colaborativo. En la figura 4 se muestran barras del estudio. 


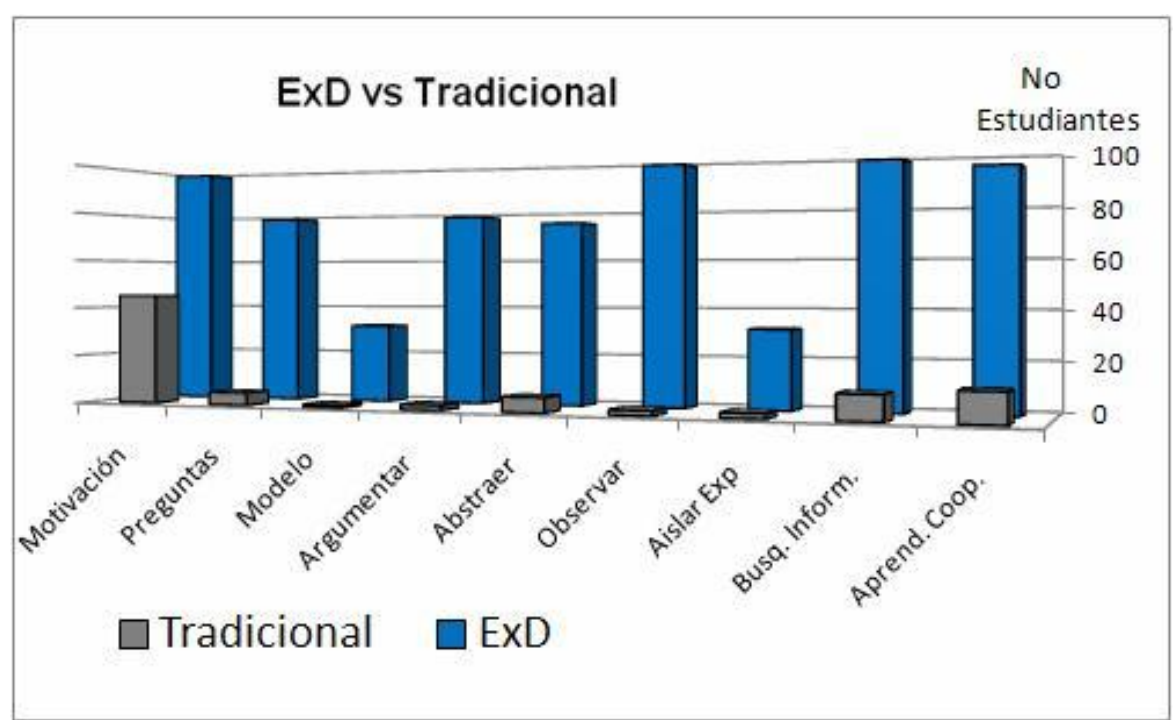

Figura No. 2 Se muestran las capacidades intelectivas ejercitadas o el nivel de comprensión de los dos montajes utilizados en cerca de 100 estudiantes de física mecánica durante 2006 y 2007 en tres grupos y semestres diferentes. Las barras del gráfico indican el nivel de motivación, de capacidad de preguntas, de capacidad de modelar, de capacidad de argumentar, etc, para las dos metodologías.

Se observa, por mucho, que los estudiantes lograron mejor desempeño mediante esta metodología alternativa. Sin embargo, se observa que es un poco difícil que el estudiante llegue a la modelación del fenómeno y logre un experimento controlado que refute o verifique el modelo. En realidad, llegar a esta etapa tan avanzada de abstracción, induso es difícil para el profesor:

\section{Conclusiones}

Los experimentos discrepantes pueden ser utilizados favorablemente para generar un escenario fértil y efectivo de aprendizaje de temas de la física. Un aprendizaje mediado por este tipo de montajes es fértil porque genera una postura activa del estudiante, origina necesidad de búsqueda tanto para el profesor como para el estudiante. Se observa un continuo ejercicio de capacidades intelectivas como observar, interrogar, conjeturar, analizar, abstraer, modelar y en consecuencia un desarrollo continuo de la intuición física del estudiante.

Falta aún caracterizar las etapas, si las hubiera, por las que pasa el proceso cognitivo del estudiante. Desde cuando se motiva con el montaje hasta cuando modela y describe a partir de conocimientos de la física. No obstante el mayor logro, es un escenario agradable con una dinámica de pasión y fantasía en la que confluyen el maestro y el estudiante.

Actualmente estamos ejecutando un proyecto de uso de ExD en jomadas sabatinas con estudiantes de grado décimo y once del colegio CAFAM. Intentamos dilucidar si en la etapa de familiarización de un ExD, se pueden introducir conceptos de Física como una necesidad para describir el fenómeno, en cuyo caso, sería una primera aproximación hacia el aprendizaje de conceptos de la física. Caracterizar esta etapa es esencial ya que corresponde a la etapa inicial de motivación, de organización de la información para describir el fenómeno y del aprendizaje de conocimiento significativo de la Física para comprender y explicar. 


\section{Bibliografía}

Barbosa, L. H. (2008). Los experimentos discrepantes en el aprendizaje activo de la Física. Latin American Joumal of Physics Education. 2 (3), 246-252

Barbosa, L. H., Talero P. (2009). La compuerta mágica: Descripción de un flujo discrepante en dos globos elásticos interconectados. Latin American Joumal of Physics Education. 3 (1), 135-139.

Barbosa, L. H. (2003). Un movimiento discrepante en el estudio de una ley de la Física de fluidos: La eauación de Bemoulli. Revista Colombiana de Física. 35 (1), 95-98.

Barbosa, L. H. (2009). Ihbfisica. Web pág. http://hbfisica,googlepages,com/

Barrera, K. J. (2007). La enseñanza de la Física a través de habilidades investigativas: una experiencia. Latin American Joumal of Physics Education 1 (1), 39-43.

Etkina, E., Van H. A., Brookes, D. T., Mills, D. (2002). Role of experiments in physics instruction - an approach. Physics. Teacher. 40 (6), 351-355. Press.

Festinger, L. (1957). A theory of cognitive dissonance. Stanford, New York: Stanford University

Heisenberg, W. (1975). La imagen de la naturaleza. Barcelona, España: Editorial Muy Interesante.

Hestenes, D. (2006). Notes for a Modeling Theory of Science, Cognition and Instruction. Arizona, EEUU: Modeling Instruction Program. Arizona State University.

Hofstadter, D. R. (2003). Gödel, Escher, Bach: Una etema trenza dorada México D.F., México: Consejo Nacional de Ciencia y Tecnología.

Krauss, L. M. (1996). Miedo a la Física: Una guía para perplejos. Santiago, Chile: Editorial Andrés Bello.

Liem, T. L (1991). Invitations to Science Inquiry, California, EEUU: Science Inquiry Enterprises.

Redish, E. F., Saul, J. M, y Steinberg, R. N. (1997). On the effectiveness of active-engagement microcomputer-based laboratories. Am. J. Phys. 65, 45-54.

Redish, E. (1998). Millikan Award Lecture Building a Science of Teaching Physics, Am. J. Phys. 67. 562-573.

Talero, P., Barbosa, L.H. (2009). Botellas equilibristas: Extracción discrepante de un billete desde la boca de dos botellas verticales invertidas. Latin American Joumal of Physics Education. 3 (2), 433-438. 